\title{
Spectral Characteristics of the 2dFGRS-NVSS Galaxies
}

\author{
C. A. Jackson ${ }^{1}$ and D. M. Londish ${ }^{2}$ \\ ${ }^{1}$ Research School of Astronomy \& Astrophysics, Institute of Advanced Studies, \\ Australian National University, Canberra, ACT 0200, Australia \\ cjackson@mso.anu.edu.au \\ ${ }^{2}$ School of Physics, University of Sydney, NSW 2006, Australia \\ Received 2000 April 6, accepted 2000 August 25
}

\begin{abstract}
We have analysed the $2 \mathrm{dF}$ spectra of a sample of galaxies common to the $2 \mathrm{dF}$ Galaxy Redshift Survey (2dFGRS, Colless 1999) and the NRAO VLA Sky Survey (NVSS, Condon et al. 1998). Our sample comprises 88 galaxies selected by Sadler et al. (1999) from 30 2dFGRS fields observed in 1998. In this paper we discuss how this and future, much larger, samples of 2dFGRS-NVSS galaxies can be interpreted via analysis of those galaxies with strong narrow emission lines. Using diagnostic line ratio measurements, we confirm the majority of the eyeball classifications of Sadler et al. (1999), although many galaxies show evidence of being 'composite' galaxies-mixtures of AGN plus starburst components.
\end{abstract}

Keywords: galaxies: evolution—galaxies: jets—quasars: general—radio continuum: galaxies

\section{Introduction}

Sources common to 2dFGRS and deep radio surveys (NVSS, FIRST, SUMSS) ${ }^{1}$ comprise large samples of local, low-to-moderate power radio sources. The sheer number of objects (in the completed 2dFGRS, we expect $\sim 4000$ galaxies in common with NVSS) and the homogeneity of the spectra will allow us to make statistical studies of sub-populations among the radio sources, so revealing a much clearer picture of their properties (and perhaps identifying rare or new classes of objects). Most importantly, the overlap between 2dFGRS and NVSS provides a sample from which to derive the local radio luminosity function, as $\sim 50 \%$ of these galaxies lie at $z<$ 0.1 (Sadler et al. 1999). A future paper will derive this local radio luminosity function and discuss the contributions of individual populations. This paper reports the results of an emission-line diagnostic analysis of the preliminary sample of 2dFGRS-NVSS galaxies, checking these against the intial 'eyeball' classifications of Sadler et al. (1999).

Throughout this paper values of $H_{0}=75 \mathrm{~km} \mathrm{~s}^{-1}$ $\mathrm{Mpc}^{-1}$ and $\Omega=1.0$ are used.

\section{The Sample of 2dFGRS-NVSS Galaxies}

The sample of 88 2dFGRS-NVSS galaxies has been extracted from the 2dFGRS and NVSS surveys, selecting sources whose radio-optical offset is less than 10 arcsec. Complete details of the selection criteria, crossmatching and classification procedures are described by Sadler et al. (1999). FITS files containing the reduced $2 \mathrm{dF}$ spectra for these galaxies were provided by the 2dFGRS project.

Each galaxy has an assigned spectral type of either 'S' = starburst or 'A' = AGN, defined as follows:

AGN have either a pure absorption-line spectrum, characteristic of an early-type galaxy, or an emission- line signature similar to those of optically-selected Seyferts, with dominant nebular emission lines (e.g. [OIII] and [OII]) relative to any Balmer-line emission. As Figure 1 shows, the majority of these objects have much lower radio powers than those usually associated with radio-loud AGN $\left(\log _{10}\left(P_{1.4 \mathrm{GHz}}\right) \sim 10^{21}-10^{24} \mathrm{~W}\right.$ $\mathrm{Hz}^{-1} \mathrm{sr}^{-1}$ ).

We can describe these AGN in terms of two subclasses:

The absorption-line AGN have radio powers typical of FR I radio galaxies, and optical spectra typical of early-type host galaxies. These sources show none of the characteristic emission lines associated with star formation processes.

The emission-line AGN could be (i) 'radio-quiet' Seyfert 2s-late-type hosts with sub-pc-scale radio corejet structures, (ii) emission-line FRI radio galaxies-rare but not unknown, or (iii) emission-line FR II radio galaxies, although this is unlikely as these are usually more powerful, $\log _{10}\left(P_{1.4 \mathrm{GHz}}\right) \geq 10^{24} \mathrm{~W} \mathrm{~Hz}^{-1} \mathrm{sr}^{-1}$, and are rare at $z<0.3$.

Starburst galaxies have emission-line signatures similar to those of optically-selected star-forming galaxies, with strong Balmer-line emission relative to any other emission features. Figure 2 shows that most of these objects are of low radio powers, $\log _{10}\left(P_{1.4 \mathrm{GHz}}\right) \leq 10^{22}$ $\mathrm{W} \mathrm{Hz}{ }^{-1} \mathrm{sr}^{-1}$, typical of known starburst galaxies.

Our sample comprises 36 starburst galaxies and 52 AGN. At the time of this analysis, FITS data were unavailable for 3 of the 36 starburst galaxies. Of the remaining 33, 22 have both $\mathrm{H} \alpha$ and $\mathrm{H} \beta$ in emission within the 2dFGRS wavelength range (i.e. they lie at $z<$ 0.2 ). The $52 \mathrm{AGN}$ can be subdivided into one broad-line Seyfert 1, 40 absorption-line (early-type) galaxies, one galaxy at $z>0.2$, and 10 with both $\mathrm{H} \alpha$ and $\mathrm{H} \beta$ in emission (and $z<0.2$ ). The 22 starburst galaxies and 10

1 NVSS: the NRAO VLA Sky Survey at $1.4 \mathrm{GHz}$, Condon et al. (1998); FIRST: Faint Images of the Radio Sky at $20 \mathrm{~cm}$, White et al. (1997); SUMSS: the Sydney University Molonglo Sky Survey at 843 MHz, Bock, Large \& Sadler (1999). 

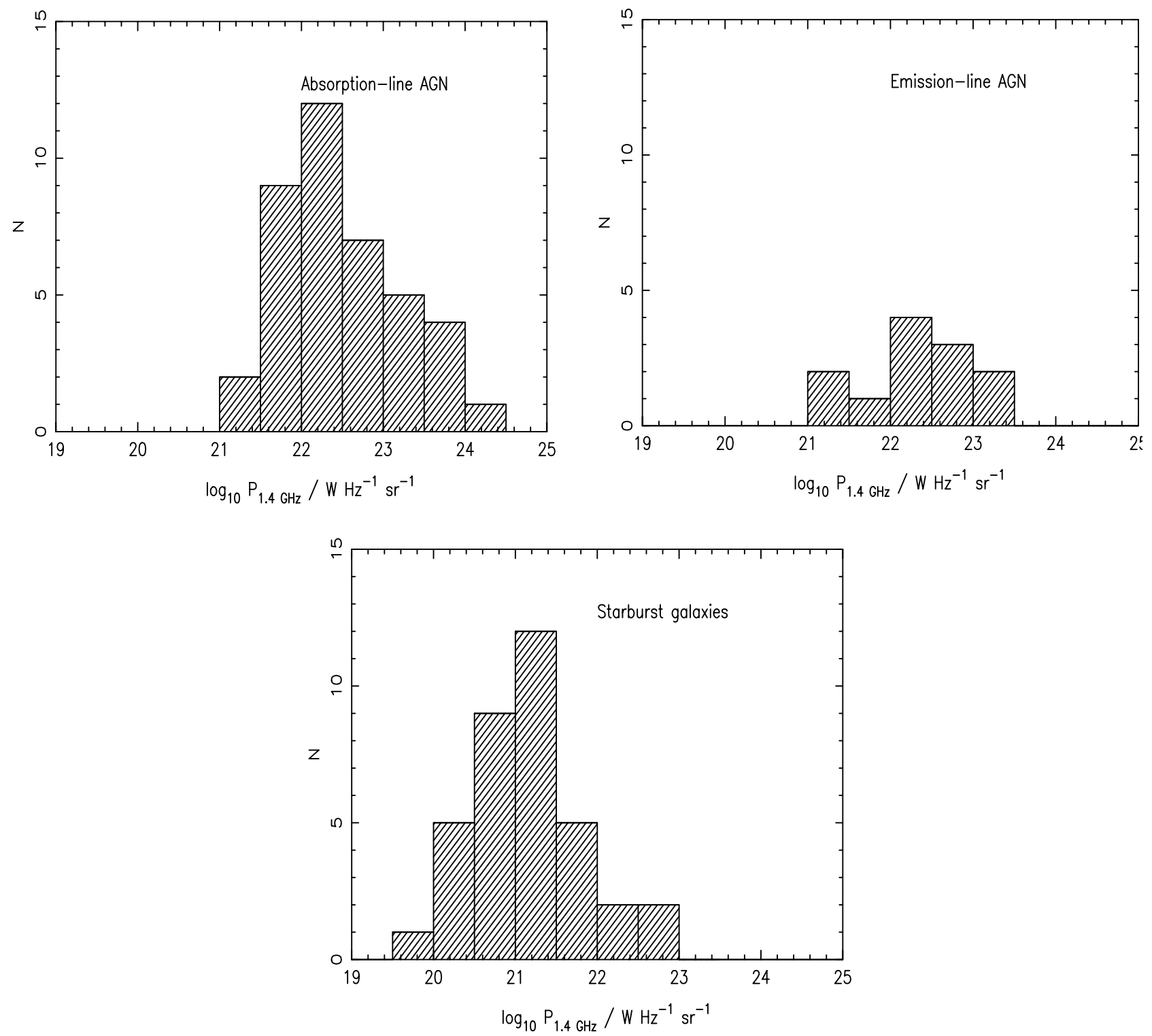

Figure 1-Luminosity distributions by type for the 88 2dFGRS-NVSS galaxies.

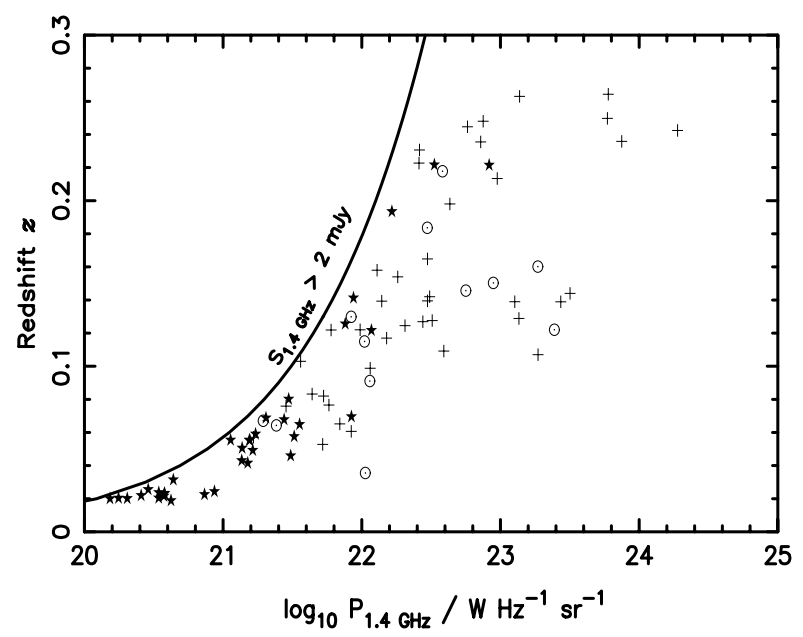

Figure 2-Radio power as a function of redshift for the sample of 88 2dFGRS-NVSS galaxies. Symbols show the initial galaxy classification types: $\star=$ starburst galaxy, $\odot=$ emission-line AGN, $+=$ absorption-line AGN.

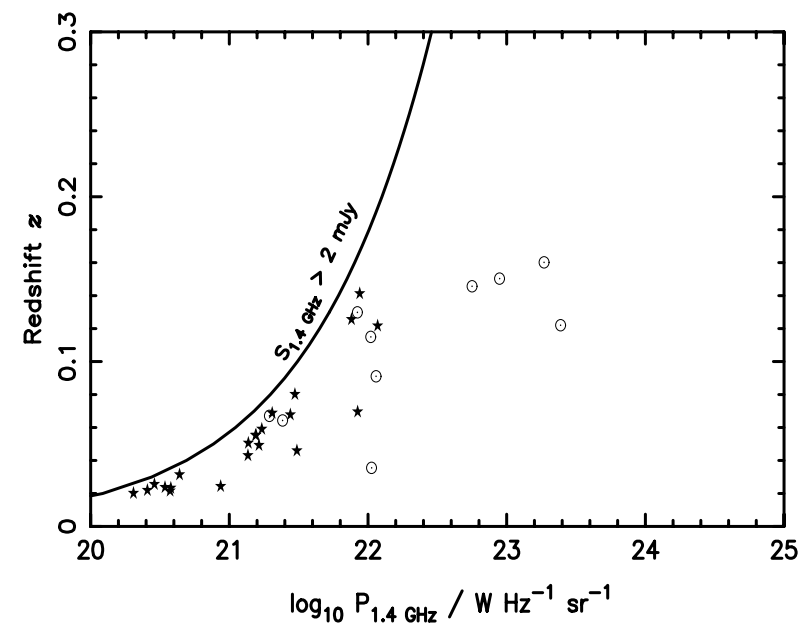

Figure 3-Radio power as a function of redshift for the 32 narrowemission-line 2dFGRS-NVSS galaxies. Symbols show the initial galaxy classification types: $\star=$ starburst galaxy, $+=$ emission-line AGN. 

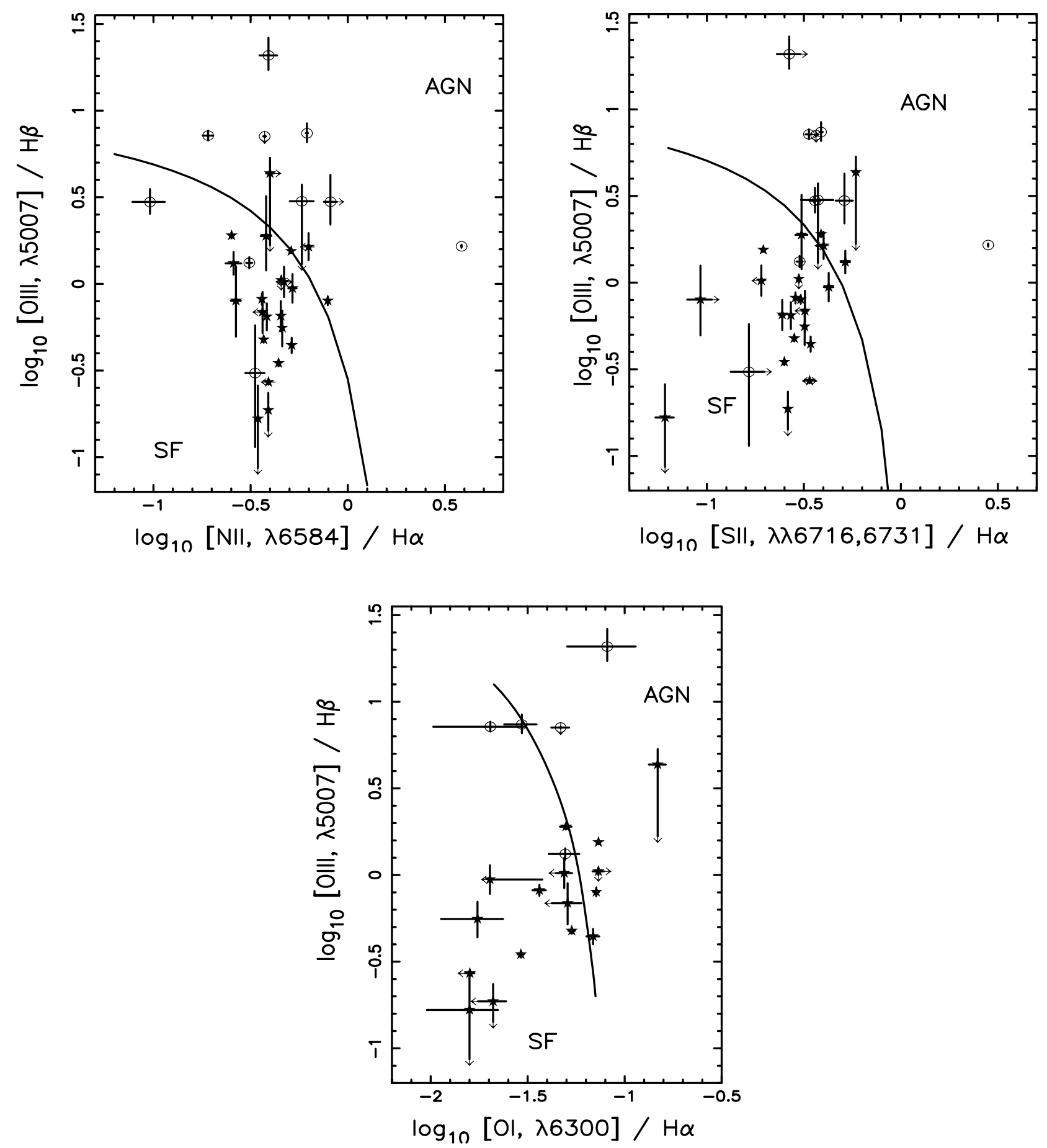

Figure 4-Diagnostic emission-line diagrams for the narrow-line galaxy sample. The symbols reflect the spectral types assigned by Sadler et al. (1999): $\odot=$ AGN, $\star=$ starburst. The solid curves in the [NII] and [SII] plots are from Kewley et al. (2000), whilst the solid curve in the [OI] plot is from Veilleux \& Osterbrock (1987). These curves divide non-thermal emission-line characteristics ('AGN') from thermal ('starburst galaxies', characterised by OB stars). Error bars have been calculated from the S/N ratio in each spectrum and the effects of residual night-sky features. Upper and lower limits are marked with arrows.

AGN with Balmer-line emission form our 'narrow-line galaxy sample'.

Figure 2 shows the distribution of radio power with redshift for the sample of galaxies. The starburst galaxies cluster at low redshifts due to the flux density limit of the NVSS. The AGN generally lie at higher redshifts and radio powers. The highest-power objects are absorption-line AGN.

\section{Emission-line Diagnostic Analysis}

We determine the emission-line ratios for the narrowline galaxy sample using the lines of $\mathrm{H} \alpha, \mathrm{H} \beta$, [OIII], [NII], [SII] and [OI]. Figure 3 shows the distribution of radio powers and redshifts for this sample. Comparing this distribution to those in Figure 1, we find we have an unbiased $(P-z)$ subset of the complete sample. 
Table 1. Emission-line ratios

\begin{tabular}{|c|c|c|c|c|c|c|c|}
\hline \multirow{2}{*}{$\begin{array}{l}\text { 2dFGRS } \\
\text { Galaxy ID }\end{array}$} & \multirow{2}{*}{$b_{J}$} & \multirow[t]{2}{*}{ Redshift } & \multirow{2}{*}{$\log _{10}\left(P_{1.4 \mathrm{GHz}}\right) \dagger$} & \multicolumn{4}{|c|}{ Measured line ratios } \\
\hline & & & & {$[\mathrm{OIII}] / \mathrm{H} \alpha$} & {$[\mathrm{NII}] / \mathrm{H} \alpha$} & {$[\mathrm{SII}] / \mathrm{H} \alpha$} & {$[\mathrm{OI}] / \mathrm{H} \alpha$} \\
\hline \multicolumn{8}{|c|}{ Initial classification $\mathrm{SF}$} \\
\hline TGS166Z108 & 17.2 & 0.0801 & 21.5 & $0.477 \pm 0.020$ & $0.369 \pm 0.003$ & $0.282 \pm 0.003$ & $0.053 \pm 0.002$ \\
\hline TGS206Z164 & 14.6 & 0.0255 & 20.5 & $1.887 \pm 1.324$ & $0.380 \pm 0.024$ & $0.307 \pm 0.023$ & \\
\hline TGS206Z015 & 17.4 & 0.0685 & 21.9 & $1.544 \pm 0.040$ & $0.511 \pm 0.002$ & $0.195 \pm 0.002$ & $0.073 \pm 0.002$ \\
\hline TGS232Z060 & 16.8 & 0.0590 & 21.2 & $0.443 \pm 0.046$ & $0.514 \pm 0.007$ & $0.342 \pm 0.007$ & $0.069 \pm 0.005$ \\
\hline TGS235Z125 & 14.8 & 0.0243 & 20.9 & $<0.167 \pm 0.093$ & $0.345 \pm 0.008$ & $0.061 \pm 0.007$ & $0.016 \pm 0.006$ \\
\hline TGS236Z095 & 17.6 & 0.1420 & 20.6 & $<0.187 \pm 0.048$ & $0.390 \pm 0.005$ & $0.262 \pm 0.004$ & $<0.021 \pm 0.003$ \\
\hline TGS236Z091 & 17.0 & 0.0552 & 21.2 & $0.687 \pm 0.210$ & $<0.364 \pm 0.012$ & $<0.320 \pm 0.011$ & $<0.051 \pm 0.009$ \\
\hline TGS236Z194 & 15.5 & 0.0237 & 20.5 & $0.557 \pm 0.144$ & $0.459 \pm 0.009$ & $0.319 \pm 0.008$ & $0.017 \pm 0.006$ \\
\hline TGS238Z047 & 16.5 & 0.0215 & 20.6 & $0.271 \pm 0.009$ & $<0.391 \pm 0.001$ & $0.338 \pm 0.001$ & $0.016 \pm 0.001$ \\
\hline TGS238Z180 & 17.2 & 0.1255 & 21.9 & $0.942 \pm 0.197$ & $0.519 \pm 0.026$ & $0.424 \pm 0.024$ & $<0.020 \pm 0.017$ \\
\hline TGS239Z196 & 14.8 & 0.0219 & 20.4 & $0.798 \pm 0.455$ & $0.266 \pm 0.016$ & $>0.093 \pm 0.014$ & \\
\hline TGS318Z156 & 18.3 & 0.0687 & 21.3 & $0.799 \pm 0.047$ & $0.789 \pm 0.004$ & $0.305 \pm 0.003$ & $0.071 \pm 0.002$ \\
\hline TGN218Z230 & 15.9 & 0.0201 & 20.3 & $0.654 \pm 0.142$ & $0.452 \pm 0.011$ & $0.245 \pm 0.010$ & \\
\hline TGN220Z065 & 17.3 & 0.0492 & 21.2 & $<4.340 \pm 1.000$ & $>0.398 \pm 0.018$ & $0.586 \pm 0.021$ & $0.148 \pm 0.015$ \\
\hline TGN222Z132 & 16.7 & 0.0505 & 21.1 & $1.633 \pm 0.329$ & $<0.628 \pm 0.009$ & $<0.400 \pm 0.008$ & \\
\hline TGN231Z143 & 15.5 & 0.0314 & 20.6 & $0.647 \pm 0.124$ & $0.383 \pm 0.007$ & $0.271 \pm 0.007$ & \\
\hline TGN239Z061 & 17.5 & 0.0554 & 21.2 & $1.902 \pm 0.076$ & $0.252 \pm 0.004$ & $0.387 \pm 0.005$ & $0.050 \pm 0.003$ \\
\hline TGN239Z082 & 18.8 & 0.1412 & 21.9 & $<1.050 \pm 0.054$ & $0.455 \pm 0.007$ & $0.298 \pm 0.006$ & $>0.073 \pm 0.005$ \\
\hline TGN239Z221 & 17.4 & 0.0430 & 21.1 & $0.349 \pm 0.015$ & $0.440 \pm 0.002$ & $0.251 \pm 0.001$ & $0.029 \pm 0.001$ \\
\hline TGN239Z172 & 16.2 & 0.0459 & 21.5 & $1.026 \pm 0.229$ & $0.470 \pm 0.007$ & $<0.191 \pm 0.005$ & $<0.049 \pm 0.005$ \\
\hline TGN240Z019 & 19.3 & 0.1216 & 22.1 & $1.314 \pm 0.216$ & $0.258 \pm 0.024$ & $0.517 \pm 0.029$ & \\
\hline TGN309Z233 & 17.9 & 0.0677 & 21.4 & $0.817 \pm 0.062$ & $0.361 \pm 0.004$ & $0.286 \pm 0.004$ & $0.036 \pm 0.003$ \\
\hline \multicolumn{8}{|c|}{ Initial classification AGN } \\
\hline TGS207Z011 & 14.2 & 0.0355 & 22.0 & $1.649 \pm 0.035$ & $3.845 \pm 0.014$ & $2.817 \pm 0.011$ & $0.553 \pm 0.004$ \\
\hline TGS218Z173 & 18.6 & 0.0911 & 22.1 & $7.170 \pm 0.499$ & $0.191 \pm 0.012$ & $>0.335 \pm 0.013$ & $0.020 \pm 0.010$ \\
\hline TGS220Z128 & 18.9 & 0.1298 & 21.9 & $20.817 \pm 5.495$ & $0.391 \pm 0.042$ & $>0.265 \pm 0.038$ & $0.081 \pm 0.033$ \\
\hline TGS233Z084 & 18.4 & 0.0643 & 21.4 & $2.972 \pm 0.554$ & $0.096 \pm 0.019$ & $0.361 \pm 0.023$ & \\
\hline TGS234Z066 & 18.9 & 0.1503 & 22.9 & $<7.101 \pm 0.098$ & $0.373 \pm 0.006$ & $0.366 \pm 0.006$ & $0.047 \pm 0.005$ \\
\hline TGS313Z081 & 17.7 & 0.1220 & 23.4 & $0.306 \pm 0.271$ & $0.334 \pm 0.039$ & $>0.165 \pm 0.034$ & \\
\hline TGS313Z100 & 17.1 & 0.1457 & 22.8 & $<3.000 \pm 0.741$ & $0.580 \pm 0.086$ & $0.374 \pm 0.075$ & \\
\hline TGN231Z211 & 16.4 & 0.0671 & 21.3 & $7.402 \pm 1.041$ & $0.616 \pm 0.009$ & $0.388 \pm 0.008$ & $0.029 \pm 0.006$ \\
\hline TGN239Z017 & 18.4 & 0.1150 & 22.0 & $2.976 \pm 1.282$ & $>0.814 \pm 0.064$ & $0.511 \pm 0.054$ & \\
\hline TGN238Z202 & 19.3 & 0.1602 & 23.3 & $1.323 \pm 0.103$ & $0.311 \pm 0.011$ & $0.301 \pm 0.011$ & $0.049 \pm 0.009$ \\
\hline
\end{tabular}

$\dagger$ Units $\mathrm{W} \mathrm{Hz}^{-1} \mathrm{sr}^{-1}$

Even though the 2dFGRS spectra are not fluxcalibrated, the pairs of emission lines were originally chosen to be close in wavelength. The line ratios are therefore accurately determined even for our uncalibrated spectra. The flux in each emission line is measured using the NOAO IRAF splot routine, fitting a Gaussian function to each emission feature. Diagnostic line ratios for $[\mathrm{NII}] \lambda 6583 / \mathrm{H} \alpha,[\mathrm{SII}] \lambda \lambda 6716,6731 / \mathrm{H} \alpha$ and $[\mathrm{OI}] \lambda 6300 / \mathrm{H \alpha}$ are plotted in Figure 4 and the data summarised in Table 1.

The diagnostic diagrams reveal that the majority of our narrow-line galaxy sample have [NII] $\lambda 6583 / \mathrm{H} \alpha$, [SII] $\lambda 6716+\lambda 6731 / \mathrm{H} \alpha$ and [OI] $\lambda 6300 / \mathrm{H} \alpha$ ratios that straddle the region dividing AGN and starburst galaxies. 
Table 2. Emission-line ratio classifications

\begin{tabular}{|c|c|c|c|c|c|c|}
\hline \multirow{2}{*}{$\begin{array}{l}\text { 2dFGRS } \\
\text { Galaxy ID }\end{array}$} & \multirow{2}{*}{$\operatorname{Mag} b_{J}$} & \multirow[t]{2}{*}{ Redshift } & \multirow{2}{*}{$\log _{10}\left(P_{1.4 \mathrm{GHz}}\right) \dagger$} & \multicolumn{3}{|c|}{ Line ratio classification } \\
\hline & & & & {$[\mathrm{NII}] / \mathrm{H} \alpha$} & {$[\mathrm{SII}] / \mathrm{H} \alpha$} & {$[\mathrm{OI}] / \mathrm{H} \alpha$} \\
\hline \multicolumn{7}{|c|}{ Initial classification $\mathrm{SF}$} \\
\hline TGS166Z108 & 17.2 & 0.0801 & 21.5 & $\mathrm{~S}$ & S & S \\
\hline TGS206Z164 & 14.6 & 0.0255 & 20.5 & S? & S? & $\ldots$ \\
\hline TGS206Z015 & 17.4 & 0.0685 & 21.9 & $?$ & $S$ & A \\
\hline TGS232Z060 & 16.8 & 0.0590 & 21.2 & $\mathrm{~S}$ & S & $?$ \\
\hline TGS235Z125 & 14.8 & 0.0243 & 20.9 & $S$ & $S$ & $S$ \\
\hline TGS236Z095 & 17.6 & 0.1420 & 20.6 & $\mathrm{~S}$ & S & S \\
\hline TGS236Z091 & 17.0 & 0.0552 & 21.2 & S & $\mathrm{S}$ & S \\
\hline TGS236Z194 & 15.5 & 0.0237 & 20.5 & $\mathrm{~S}$ & S & $\mathrm{S}$ \\
\hline TGS238Z047 & 16.5 & 0.0215 & 20.6 & $S$ & $\mathrm{~S}$ & $S$ \\
\hline TGS238Z180 & 17.2 & 0.1255 & 21.9 & S & $\mathrm{S}$ & S \\
\hline TGS239Z196 & 14.8 & 0.0219 & 20.4 & $\mathrm{~S}$ & $\mathrm{~S}$ & $\ldots$ \\
\hline TGS318Z156 & 18.3 & 0.0687 & 21.3 & $?$ & S & A \\
\hline TGN218Z230 & 15.9 & 0.0201 & 20.3 & S & $\mathrm{S}$ & $\ldots$ \\
\hline TGN220Z065 & 17.3 & 0.0492 & 21.2 & $\mathrm{~A}$ ? & A & $\mathrm{A}$ \\
\hline TGN222Z132 & 16.7 & 0.0505 & 21.1 & A & $?$ & $\ldots$ \\
\hline TGN231Z143 & 15.5 & 0.0314 & 20.6 & S & $\mathrm{S}$ & $\ldots$ \\
\hline TGN239Z061 & 17.5 & 0.0554 & 21.2 & $\mathrm{~S}$ & $?$ & $?$ \\
\hline TGN239Z082 & 18.8 & 0.1412 & 21.9 & S & $\mathrm{S}$ & A \\
\hline TGN239Z221 & 17.4 & 0.0430 & 21.1 & S & $S$ & $S$ \\
\hline TGN239Z172 & 16.2 & 0.0459 & 21.5 & S & $\mathrm{S}$ & S \\
\hline TGN240Z019 & 19.3 & 0.1216 & 22.1 & S & A & . \\
\hline TGN309Z233 & 17.9 & 0.0677 & 21.4 & S & $\mathrm{S}$ & S \\
\hline \multicolumn{7}{|c|}{ Initial classification AGN } \\
\hline TGS207Z011 & 14.2 & 0.0355 & 22.0 & A & A & A \\
\hline TGS218Z173 & 18.6 & 0.0911 & 22.1 & A & A & $S$ \\
\hline TGS220Z128 & 18.9 & 0.1298 & 21.9 & A & A & A \\
\hline TGS233Z084 & 18.4 & 0.0643 & 21.4 & $\mathrm{~S}$ & $\mathrm{~A}$ & $\ldots$ \\
\hline TGS234Z066 & 18.9 & 0.1503 & 22.9 & $\mathrm{~A}$ & $\mathrm{~A}$ & A \\
\hline TGS313Z081 & 17.7 & 0.1220 & 23.4 & $\mathrm{~S}$ & S & $\ldots$ \\
\hline TGS313Z100 & 17.1 & 0.1457 & 22.8 & A? & A? & $\ldots$ \\
\hline TGN231Z211 & 16.4 & 0.0671 & 21.3 & A & A & $?$ \\
\hline TGN239Z017 & 18.4 & 0.1150 & 22.0 & A & A & $\ldots$ \\
\hline TGN238Z202 & 19.3 & 0.1602 & 23.3 & $\mathrm{~S}$ & $\mathrm{~S}$ & $\mathrm{~S}$ \\
\hline
\end{tabular}

$\dagger$ Units $\mathrm{W} \mathrm{Hz}^{-1} \mathrm{sr}^{-1}$

This is in contrast to optically-selected galaxy samples, whose narrow-line emission characteristics clearly delineate those with a hard ionising radiation source (AGN: right-hand side of curves in Figure 4) and those with hot OB stars (starburst, left-hand side of curves). Similar results to our line ratio distribution have been found for other radio-selected samples (e.g. Georgakakis et al. 1999) as well as IR-selected galaxy samples (Kewley et al. 2000).

In Table 2 we show how the galaxies are classified based on the individual emission-line ratio values. Where the errors associated with the line ratio span the empirical dividing line, the classification is given as $\mathrm{A}$ ? 
or S?. For the cases where the ratio lies right on the dividing line, the classification is given as ?.

We find four galaxies with different line ratio classifications to the 'eyeball' classifications of Sadler et al. (1999):

(1) TGN220Z065: Starburst galaxy, line ratio diagnostics indicate that it is an AGN. However, the $\mathrm{H} \beta$ measurement is a lower limit so that the the [OIII]/ $\mathrm{H} \beta$ value is overestimated.

(2) TGN222Z132: Starburst galaxy, line ratio diagnostics indicate that it is an AGN. However, the $\mathrm{H} \alpha$ measurement is a lower limit so that the $[\mathrm{SII}] / \mathrm{H} \alpha$ value is overestimated.

(3) TGN238Z202: AGN galaxy, line ratio diagnostics indicate that it is a starburst galaxy. Re-examining the 2dFGRS spectrum shows dominant Balmer lines characteristic of a starburst galaxy.

(4) TGS313Z081: AGN galaxy, line ratio diagnostics indicate that it is a starburst galaxy. Re-examining the $2 \mathrm{dFGRS}$ spectrum reveals strong $\mathrm{H} \alpha$, weak $\mathrm{H} \beta$ and the $5577 \AA$ line coincident with [OIII, 5007 ]. On balance this probably should be classified as a starburst galaxy.

Thus the diagnostic line ratios confirm 30 out of 32 of the 'eyeball' classifications. However, the frequency of ambiguous classifications from the line ratio diagnostics (indicated as A?, S? and ? in Table 3 below) indicates that a radio-selected sample does not clearly separate into the starburst and AGN regions defined by optically-selected samples.

Table 3. Star formation rates from IR and radio flux densities

\begin{tabular}{llll}
\hline 2dFGRS & $\log _{10}\left(P_{1.4 \mathrm{GHz}}\right) \dagger$ & \multicolumn{2}{c}{$\begin{array}{c}\text { Star formation rate } \\
\text { Galaxy ID }\end{array}$} \\
& & Radio $_{1.4 \mathrm{G}_{\mathrm{Hz}}}$ & $\mathrm{IR}_{60 \mu \mathrm{m}}$ \\
\hline TGS166Z108 & 21.5 & 9.3 & 7.1 \\
TGS206Z164 & 20.5 & 0.9 & 0.7 \\
TGS206Z015 & 21.9 & 26.4 & 77.7 \\
TGS232Z060 & 21.2 & 5.4 & 4.2 \\
TGS235Z125 & 20.9 & 2.7 & 2.1 \\
TGS236Z095 & 20.6 & 1.2 & 1.1 \\
TGS236Z091 & 21.2 & 4.9 & 2.9 \\
TGS236Z194 & 20.5 & 1.1 & 1.0 \\
TGS238Z047 & 20.6 & 1.2 & 1.3 \\
TGS238Z180 & 21.9 & 23.9 & 11.0 \\
TGS239Z196 & 20.4 & 0.8 & 0.5 \\
TGS318Z156 & 21.3 & 6.4 & 8.8 \\
TGN218Z230 & 20.3 & 0.6 & 0.6 \\
TGN220Z065 & 21.2 & 5.1 & 2.6 \\
TGN231Z143 & 20.6 & 1.4 & 1.0 \\
TGN239Z061 & 21.2 & 4.3 & 4.3 \\
TGN239Z172 & 21.5 & 9.7 & 7.4 \\
\hline
\end{tabular}

†Units $\mathrm{W} \mathrm{Hz}^{-1} \mathrm{sr}^{-1}$

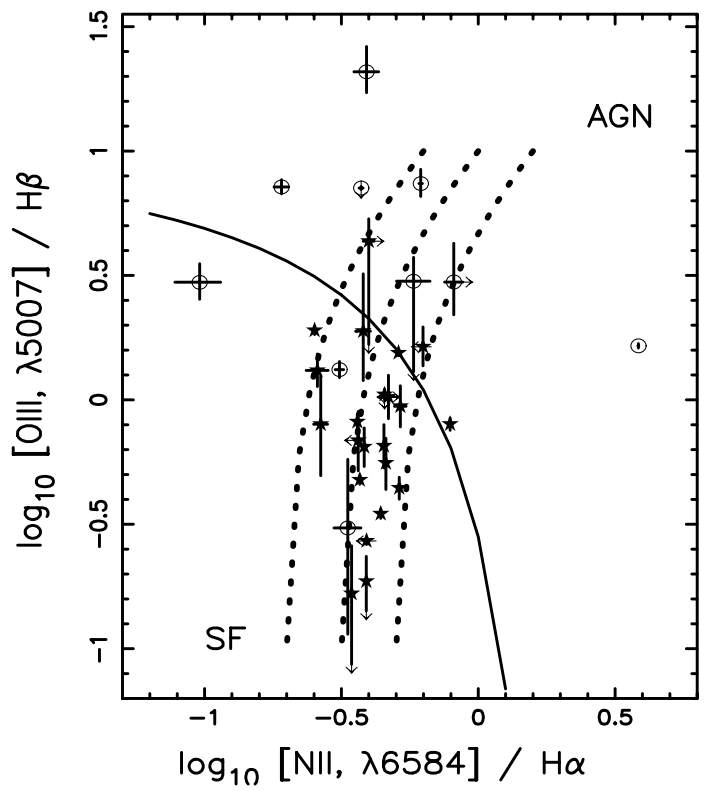

Figure 5-Diagnostic emission-line ratios for $[\mathrm{NII}] / \mathrm{H} \alpha$ as shown in Figure 4. The solid line divides the starburst (SF) and AGN sources from Kewley et al. (2000). Mixing lines are shown as dotted lines, with increasing AGN contributions lying at higher $[\mathrm{OIII}] / \mathrm{H} \beta$ values.

Further evidence as to the composite nature of the starburst galaxies can be found by comparing the starformation rates indicated by the radio and IR flux densities. Table 3 shows the flux densities and the implied star formation rates for massive stars $\left(>5 \mathrm{M}_{\odot}\right.$, Cram et al. 1998), using $60 \mu \mathrm{m}$ flux density data compiled by Sadler et al. (1999) from the IRAS Point Source and Faint Source catalogues. Two galaxies stand out as having very discrepant implied star formation rates: (1) TGS206Z015 has an IR excess, and (2) TGS238Z180 has a radio excess. In both cases the UKST IIIaJ images show that the galaxies are undergoing mergers, which is possibly enhancing or triggering starburst activity in these objects.

Furthermore, we suggest that the majority of the narrow-line galaxies whose line ratios are strung out between the two regions (star-forming and AGN) are composite galaxies: the individual line ratios lie along mixing lines whose positions on the diagnostic plots are determined by the admixture of AGN and starburst components, as shown in Figure 5.

\section{Summary}

Our analysis finds that this preliminary sample of narrow-emission-line galaxies selected from 2dFGRS and NVSS comprises a mix of starburst and AGN types. The distribution of their emission-line ratios suggests that a sizable fraction of the galaxies may be composite galaxies. Further work will determine the relative contributions of the star-forming and AGN components. 


\section{Acknowledgments}

We thank the 2dF Galaxy Redshift Survey team for advance access to their data. DML thanks the Science Foundation for Physics at the University of Sydney for a summer vacation scholarship.

\section{References}

Bock, D. C.-J., Large, M. I., \& Sadler, E. M. 1999, AJ, 117, 1578 Colless, M. M. 1999, Phil. Trans. R. Soc. Lond., 357, 105

Condon, J. J., Cotton, W. D., Greisen, E. W., Yin, Q. F., Perley, R. A., \& Broderick, J. J. 1998, AJ, 115, 1693
Cram, L., Hopkins, A., Mobasher, B., \& Rowan-Robinson, M. 1998, ApJ, 507, 155

Georgakakis, A., Mobasher, B., Cram, L., Hopkins, A., Lidman, C., \& Rowan-Robinson, M. 1999, MNRAS, 306, 708

Kewley, L., Heisler, C. A., Dopita, M. A., \& Lumsden, S. 2001, ApJ5, 132, 37

Sadler, E. M., McIntyre, V. J., Jackson, C. A., \& Cannon, R. D. 1999, PASA, 16, 247

Veilleux, S., \& Osterbrock, D. E. 1987, ApJS, 63, 295

White, R. L., Becker, R. H., Helfand, D. J., \& Gregg, M. D. 1997, ApJ, 475, 479 